\title{
In Silico Interaction and Docking Studies Indicate a New Mechanism for PML Dysfunction in Gastric Cancer and Suggest Imatinib as a Drug to Restore Function
}

\author{
Zeinab Imani-Saber, Soudeh Ghafouri-Fard*
}

\begin{abstract}
Gastric cancer as one of the most common cancers worldwide has various genetic and environmental risk factors including Helicobacter pylori (H.pylori) infection. Recently, loss of a tumor suppressor gene named promyelocytic leukemia $(P M L)$ has been identified in gastric cancer. However, no mutation has been found in this gene in gastric cancer samples. Cag A H.pylori protein has been shown to exert post transcriptional regulation of some tumor suppressor genes. In order to assess such a mechanism for PML degradation, we performed in silico analyses to establish any interaction between PML and Cag A proteins. In silico interaction and docking studies showed that these two proteins may have stable interactions. In addition, we showed that imatinib kinase inhibitor can restore PML function by inhibition of casein kinase 2.
\end{abstract}

Keywords: PML - cag A - Helicobacter pylori - casein kinase - imatinib - docking study

Asian Pac J Cancer Prev, 16 (12), 5005-5006

\section{Introduction}

Gastric cancer is the fifth most common cancer and the third leading cause of cancer death globally. Although several genetic and environmental risk factors have been identified, Helicobacter pylori (H.pylori) accounts for $60-70 \%$ of gastric cancer cases worldwide (Fock, 2014). In addition, Epstein-Barr virus (EBV) infection has been implicated in about $10 \%$ of gastric carcinomas (Sivachandran et al., 2012). Recent studies have shown decreased or loss of expression of the tumor suppressor, promyelocytic leukemia (PML) protein in gastric cancer. This event has been associated with an increased level of lymphatic invasion, higher pTNM staging, and poor prognosis (Lee et al., 2007; Kim et al., 2011). We recently have screened gastric cancer samples for $P M L$ mutations. As no deleterious mutations have been found in this gene in gastric cancer samples, it has been suggested that posttranslational modifications or protein degradation could be mechanisms by which PML is not expressed in gastric tumors (Imani-Saber et al., 2015). EBNA1 as the only EBV nuclear protein expressed in gastric cancer has been shown to disrupt PML function and consequently result in impaired responses to DNA damage and promote cell survival (Sivachandran et al., 2012). Such EBNA1 role has been demonstrated to be performed via recruiting casein kinase 2 (CK2) to PML nuclear bodies and consequent increase in CK2-mediated phosphorylation of PML proteins which triggers PML degradation (Sivachandran et al., 2010). However, the possible interaction of H.pylori proteins with PML has not been elucidated yet. Cag A H.pylori protein has been shown to induce proteasomal degradation of p53 in gastric epithelial cells infected with H.pylori (Wei et al., 2010). To evaluate the possible interaction of Cag A protein with PML, we performed In silico analyses using bioinformatics tools. In addition, we performed a docking study to asses if imatininb as a kinase inhibitor drug can influence casein kinase 2 functions.

\section{Materials and Methods}

In order to perform interaction study, pdb files corresponding to PML and Cag A proteins were retrieved from the protein data bank (PDB; http://www.rcsb.org/ $\mathrm{pdb} /$ ). Hex 8.0.0 software was used for protein docking predictions. SDF format of imatinib molecule was retrieved from PubChem (https://pubchem.ncbi.nlm.nih. gov/) and changed to PDB format using open babel 2.3.2a tool. AutoDockTools 1.5-6rc3 and AutoDock Vina were used for prediction of imatinib binding to CK2. Lysine rich residues of 71-77 in A chain of CK2 was defined as the active binding site.

\section{Results}

The total energy of interaction between Cag A and PML molecules determined by Hex 8.0.0 software in the best conformation was -466.66 which implies that such interaction would be stable (Figure 1).

The interaction energy between imatinib and CK2 
Zeinab Imani-Saber and Soudeh Ghafouri-Fard

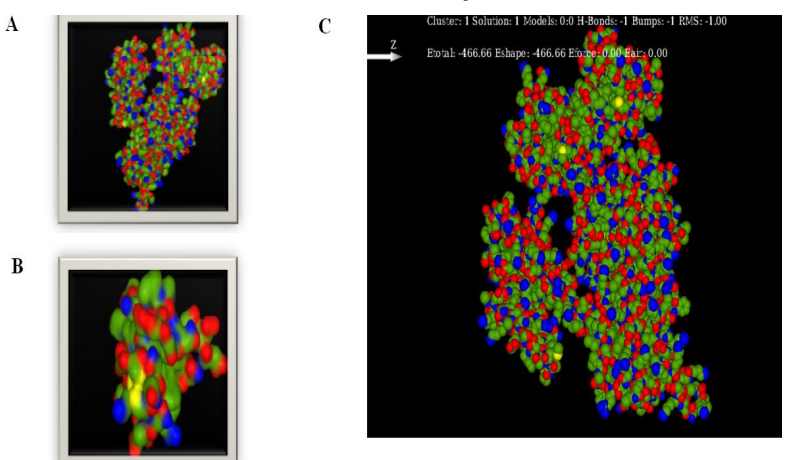

Figure 1. A. Cag A Protein Structure, B. PML Protein Structure, C. Docking Study of Cag A and PML Proteins

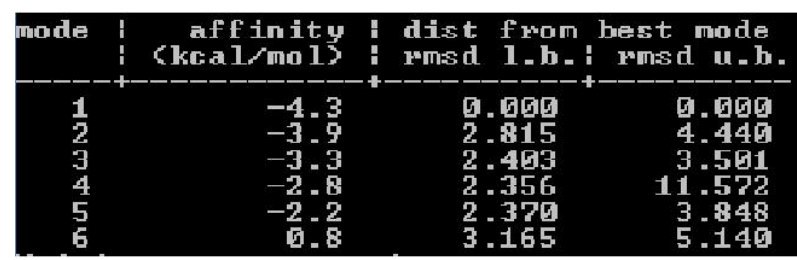

Figure 2. Affinity Values for CK2 and Imatinib Interactions

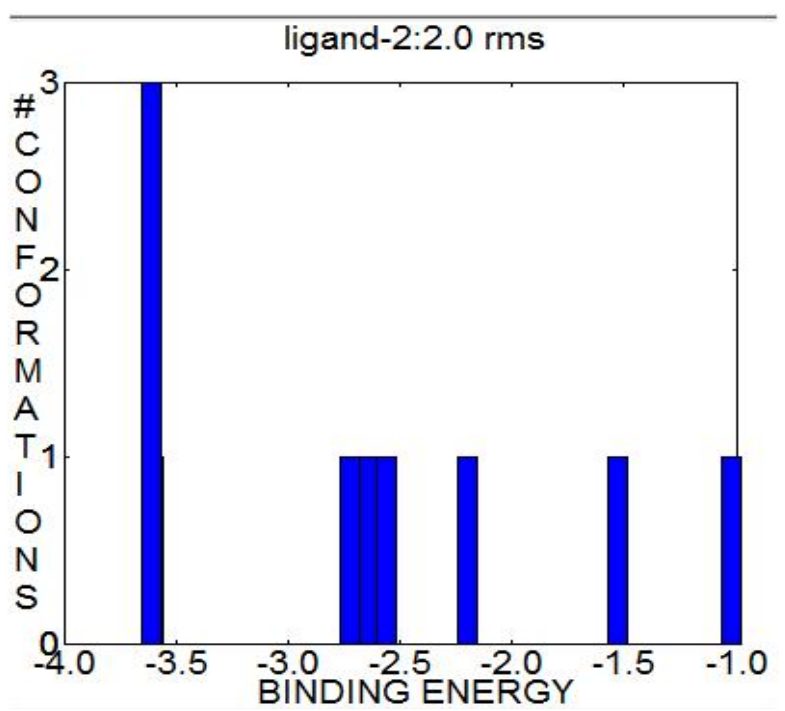

Figure 3. Clustering of Various Interactions between CK2 and Imatinib

was calculated for the Lysine 75 in the binding site. AutoDock Vina showed the values of affinity of such interactions were between -4 and $0 \mathrm{kcal} / \mathrm{mol}$ (Figure 2). Figure 3 shows the clustering of various dockings between CK 2 and imatinib which has been performed by AutoDockTools. The negative values for binding energy of these conformations indicate effective binding of these proteins at that defined binding site.

\section{Discussion}

PML has multiple functions in growth inhibition, apoptosis induction, replicative senescence, inhibition of oncogenic transformation, and suppression of migration and angiogenesis. These roles have implied that it could be a suitable therapeutic target for cancer therapy
(Imani-Saber and Ghafouri-Fard, 2014). Although multiple studies have indicated loss of PML in gastric cancer, the exact mechanism of such loss of function is not clear. Among various risk factors for gastric cancer, the role of two infections in this malignancy has been highlighted. EBV nuclear protein has been shown to interact with PML protein and degrade it. However, the data regarding interaction of H.pylori proteins with PML is missing. We have performed In silico interaction study to find whether Cag A protein could interact with PML. Such studies showed that these two proteins have stable interactions. Furthermore, to find an effective molecule to restore PML function, we analyzed the interaction of the famous kinase inhibitor imatinib with CK2 which has a fundamental role in PML degradation. Our In silico studies suggest that imatinib can be served as a drug to inhibit CK2 function and maintain PML nuclear bodies. However, further researches are needed to confirm the results of such studies.

\section{References}

Fock KM (2014). Review article: the epidemiology and prevention of gastric cancer. Aliment Pharmacol Ther, $\mathbf{4 0 ,}$ 250-60.

Imani-Saber Z, Ghafouri-Fard S (2014). Promyelocytic leukemia gene functions and roles in tumorigenesis. Asian Pac J Cancer Prev, 15, 8021-8.

Imani-Saber Z, Yousefi-Razin E, Javaheri M, et al (2015). PML gene mutations do not contribute in gastric adenocarcinoma. Asian Pac J Cancer Prev, [Epub ahead of print].

Kim HJ, Song DE, Lim SY, et al (2011). Loss of the promyelocytic leukemia protein in gastric cancer: implications for IP-10 expression and tumor-infiltrating lymphocytes. PLoS One, 6, 26264.

Lee HE, Jee CD, Kim MA, et al (2007). Loss of promyelocytic leukemia protein in human gastric cancers. Cancer Lett, 247, 103-9.

Sivachandran N, Cao JY, Frappier L (2010). Epstein-Barr virus nuclear antigen 1 Hijacks the host kinase CK2 to disrupt PML nuclear bodies. $J$ Virol, 84, 11113-23.

Sivachandran N, Dawson CW, Young LS, et al (2012). Contributions of the Epstein-Barr virus EBNA1 protein to gastric carcinoma. $J$ Virol, 86, 60-8.

Wei J, Nagy TA, Vilgelm A, et al (2010). Regulation of p53 tumor suppressor by Helicobacter pylori in gastric epithelial cells. Gastroenterol, 139, 1333-43. 